\title{
Art Inheritance: An Education Course on Traditional Pattern Morphological Generation in Architecture Design Based on Digital Sculpturism
}

\author{
Chong Xu ${ }^{1,2, *}$, Yi Huang ${ }^{2,3}$ and Bart Dewancker ${ }^{2}$ (D) \\ 1 College of Architecture and Urban Planning, Qingdao University of Technology, Qingdao 266033, China \\ 2 Graduate School of Environmental Engineering, University of Kitakyushu, Kitakyushu 808-0135, Japan; \\ huangyi425@126.com (Y.H.); bart@kitakyu-u.jp (B.D.) \\ 3 Department of Mechanical and Electrical Engineering, Qingdao Agricultural University, \\ Qingdao 266109, China \\ * Correspondence: xuchong621@hotmail.com
}

Received: 6 March 2020; Accepted: 25 April 2020; Published: 6 May 2020

\begin{abstract}
Cultural communication and art heritage represent a repository of highly condensed information of past time that depends not only on the expansion of emerging digital media but also on the transformation of language and knowledge. In this course, students try to extract single elements from the traditional Chinese cultural patterns and redesign them, then combine them into existing buildings, attempting to balance the coherence, heterogeneity of space, and the uniqueness of projects. It provides favorable conditions in the new era for the traditional patterns to be re-activated. It is a combination of technology and art. The purpose of this course is not to discover new theories or new technologies but to provide morphological possibilities under the existing digital techniques in cultural symbols. We try to build new ideas and innovative digital expressions for traditional Chinese patterns, realize the collision of culture and technology from experimental trials on the existing architectures in other unimagined forms. It is not only the research reproduction of traditional patterns but also brings new vitality and fresh ideas for architecture design.
\end{abstract}

Keywords: art inheritance; digital sculpturism; traditional pattern; culture identification; digital arts

\section{Introduction}

"What is history? An echo of the past in the future, a reflex from the future on the past."-Victor Hugo [1].

Human beings are living in this era, surrounded by wireless communication and digital information. It is an innovating time of recognizing space, time, and material.Technology has changed the original mode of culture, society, and all aspects of life, even the relationship between people because of the intervention of "digital". The combination of digital technology and architectural design has achieved the leap progress of the design from the original computer-assisted drawing to the computer-assisted designing. Design forms the thinking mode of multidisciplinary integration. Digital technology, as a new solution and a new tool in global architecture, is attempting to inject new vitality into the classical elements.

The word "sculpturism" in the title first appeared in the evaluation of Frederick Kiesler's project "The Endless House". Lars Spuybroek had his own opinion: Sculpturism was the synonym of Beaux-Arts schools of design. It aimed to rethink and redefine the technology in combination with feeling, material, and structure [2]. He wanted to stay far away from sculpturism [3] because it means "favors form over function" and seeks to adhere to the aesthetic values of other works and disciplines [4]. In some publications, editors use the word "sculpturism" to describe the influential 
architecture, including the projects by LA legends Eric Owen Moss, Thom Mayne, and a host of emerging talents [5]. In the early times, architect Frank Gehry developed a very highly specific visual language that determines all his projects. His success was remarkable. People began to realize the potential of digital software [6]. In the 20th century, Lebbeus Woods was classified as a different kind of architect by the mainstream architecture circles at that time, because of his extremely unconventional heterotopia creativity and astonishing architectural form like high-houses [7]. In the 21st century, Zaha Hadid created a personalized architectural language with advanced digital design tools. Her projects became the most sculptural and beautiful [8]. In recent years, Professor Hernan Diaz Alonso (an Argentine-American architect, professor in SCI-Arc in Los Angeles) pushed the "sculpturism" to an extreme degree. He commented on the cases in his book: They are grotesque, not ugly. They are mannered, not baroque. They are the products of an excellent eye. They are projects that look a whole lot less like conventional architecture. They seem to have undergone an amazing process of mutation that has made them big and hard [9]. The idea of "digital sculpturism" allows the designers to focus on the artistic and expressive possibilities of arts and architecture. The richness details of unique and potent elements are the reflection of "excitement, enthusiasm, energy and high spirits" [10]. In this aesthetic way, precise visual effects become the primary consideration. The design principles and methods presented by "digital sculpturism" emphasize the sculptural expressiveness in object forms. The form provides the conditions for the seamless connection between reality and virtuality, and less attention to functions, which is conducive to the poetic expression of modeling. Many iterations models were written by digital software, with the complex system attached to the original structure, which was eventually applied to architectural design. The new digital technology can not only strengthen the impact of visual architectural form but also can make the design more ethnic, aesthetic sense, more stylish.

\section{Background}

Chinese traditional patterns mainly refer to symbolic meanings created in the birth and development process of Chinese civilization. They represent the aesthetic taste and value orientation of every period. It is inevitable to brand with the mark of era culture. In the early time, the traditional patterns originated from nature. The beauty of them came from the natural form. After continuous simplification, the models became refined and represented a very close relationship between geometry and mathematics. "Harmony of form consists in the proper balancing and contrast of the straight, the inclined, and the curved." [11]. "All junctions of curved lines with curved, or of curved lines with straight, should be tangential with each other." [11]. For the Chinese patterns, triangulation is the main feature, the geometrical arrangement is absolute and undisguised, but softened by free treatment of the intermediate spaces left by the triangulation [12]. Of course, the patterns are the symbol of images of languages, the pictorial expression of words. Thus, they have a close relationship with the written words. It is an essential branch of the language symbol system. The Chinese character symbol system does not only simulate the object of nature but can engrave the continuity of cultural communication.

Traditional patterns play an essential role in the conventional design. First, it provides abundant resources for design. In the long history of patterns, its symbolic and artistic conception becomes a unique form. The original study of pattern logic does not originate from the design but the kaleidoscope. Scottish scientist and inventor David Brewster was one of the early scholars in this area. The research on the mathematical logic behind the pattern graphics appeared in the kaleidoscope. David revealed the different levels hidden in the pattern with simple line drawing, brought some elementary concepts of geometry into play to create an attractive context [13]. A deep mathematical foundation also supported the patterns created by Islamic craftsmen in the workshop. More than a hundred designs, all based on a simple schema, are presented in "The Power of Form Applied to Geometric Tracery" [14]. George Bain described how mathematical analysis can be used to reorganize and understand Celtic knots as essential representatives of traditional British culture. The fabricated structure was based on a three-grid system [15]. Fractal mathematics also has a significant impact on the remodeling of 
patterns [16]. In terms of construction, vegetal motifs, and geometric patterns were gradually applied in Islamic art and architecture under the influence of ancient culture [17]. Geometry patterns based on the mathematical division became the most prominent decorative system. They were linking woven designs to a mesh system [18]. It played an important role in the structural and decorative elements of traditional Persian architectures [19]. The author tried to solve 3D problems through 2D patterns. The solution was to generate new intricate patterns by using simple existing components or modules, refining, and defining criteria for Muqarnas classification. In the twentieth century, many famous architects expressed their opinion on ornaments and patterns, like Adolf Loos's famed theory "ornament and crime", whose real meaning is imagining an aesthetic liberated from ornament. He did not lose his faith in ornament but hoped to make and use new patterns and new decorations [20]. Under "digital technology", the traditional model has more possibilities and openness. Professor Picon proposed that architects should refocus on the surface and facade because of the rediscovery of the patterns, the development of the digital technology [21]. He also defined the contemporary trend, pointing out that "the reinvention of ornament" is better than "the return of ornament" [22]. The key to development is the historical analysis, rather than "bringing back", there is no pure utilization of past things [23]. Therefore, in the digital age, the application of ornament in contemporary architecture is grounded on the masterly use of computer-aided design and manufacturing programs. In this sense, ornament today has become creative, rather than the retrospective element in which architects reinterpret, design, and fabricate their own volition [24]. The research on the traditional pattern in China was initially from Lin Huiyin, who discussed the influence of Indian Buddhist art in the Western Regions and the exchanges and collisions between China and the Western countries in terms of architecture, sculpture, and patterns in 1933 [25]. Linxun conducted an in-depth analysis of the two-dimensional pattern field, systematically summarized the periodic patterns and quasi-periodic patterns, and proposed detailed two-dimensional illustrations [26]. Due to the limitations of technology, aesthetic and artistic aspects of traditional Chinese patterns are only presented by the two-dimensional spatial showing, such as form and color, with merely making up and piling up, little innovation. It is not the real inheritance and reuse of traditional patterns.

In terms of combining pattern graphics and digital technology, designers are studying the "free interface" of digital technology, looking forward to continuous innovation and leading fashion. From digital printing and fine carving technology to 3D printing, light-sensitive patterns, and more, traditional models exist in different forms of innovative thinking. Designers try to deepen their thinking and convey the spirit and connotation of conventional designs with different visual aesthetics. The digitization of the pattern makes the projects achieve extraordinary degrees of freedom. The system's automatic generation mode and designer's recreation mode have substantial autonomy. Digital patterns can be derived from $2 \mathrm{D}$ to $3 \mathrm{D}$ and even $4 \mathrm{D}$, according to different pieces of computer software, such as holographic stereo imaging technology and virtual reality technology. Shanghai Pioneer Design Co. Ltd. XUBERANCE has opened up a brand-new aesthetic design method to interpret and revive traditional Chinese culture. They try to reinterpret it with new technology. The project: "Viewing the Clouds", inspired by Liu Yuxi's masterpiece (a Chinese poet, philosopher, and essayist, 772 842) and named the entire series of works with the expression of clouds. The patterns of Chinese classical garden decoration present a different modern aesthetic concept through 3D printing technology [27].

Zaha Hadid Architects introduced a new experimental architecture during the annual Milan Design Week as part of the City Show. The project is called "Thallus" [28], which means that the stems and leaves of the plant are not distinguished in Greek. Designer Benjamin Dillenburger and Michael Hansmeyer used the algorithmic design method and 3D printers to complete an extremely complex object: The Arabesque wall. The project has an incredible form, and its complexity is a new challenge for people's perception ability. The designers explained: Architecture should be surprising, exciting, and irritating. They used full 3D printing technology to turn seven tons of sand and gravel into a fantasy cave. Through the calculation of up to 156GB data volume, they finally formed a 3D printed work composed of 1.35 million faces. The work presents a porous, multi-layered texture with a 
seemingly endless mysterious beauty, from simple to complex. It shows how the ability of geometric space expression under digital technology makes beautiful architecture from conception to reality. It abandons the traditional paradigm of rationalization and standardization. The new architectural concept emphasizes the viewer's understanding and perception, inspiring the viewer's sense of joy, curiosity, and confusion [29].

Numerous studies have shown that it is incomparable between pattern art and many other art forms in terms of the long history and scope. Its existence provides a rich source of value for design. With the emergence and penetration of new materials, new processes, and new technologies in different periods, new developments in design will re-innovate based on traditional forms and meanings. Education is of utmost importance for the future of sustainable development, especially in the field of architecture. It is a key factor in the reorientation of the design industry towards a system of buildings and cities [30]. Architectural design, as one of the most complicated negotiation processes and globally recognized cultural practices, is both an academic topic and a career direction. The design should be comprehensive, including all technology and aesthetics, as well as all technical, aesthetic, political, and economic issues in a particular society.

In this regard, this paper aims to break the traditional perception of two-dimensional graphics through advanced digital concepts and abstract graphical thinking. The design incorporates pattern diagrams into the architectural thinking system, elaborates complex processes in simple abstract forms, and directly adopts the generative nature of diagrams. Under the "digital sculpturism thinking", the designer explores innovative ways to design, more modern, more visual impact. Besides them, it also shows its vitality and freedom, seeks more possibilities, more diversified prototypes based on elements with the aesthetic value and social diversification. Try efforts to trace back the origin of the design, the origin of culture, the emphasis of practice is to refine and apply traditional patterns. The new digital solution can not only strengthen the impact of visual architectural form but also can make the design more extreme, more enthusiastic, with more details. The characteristics of the times can also be endowed with more traditional elements to complete the reconstruction and extension of the traditional patterns. In addition to two-dimensional decoration, we try to present it in a three-dimensional digital sculpting form, providing creative transformation and innovative development for the expression of traditional culture. We also hope that people can pay more attention to traditional pattern culture and increase people's cultural identity and self-confidence.

\section{Materials and Methods}

\subsection{A Pedagogical Framework for the Course}

The course is developed for students in the sophomore year who wish to engage with emerging techniques in design. It offers new means to design inquiry through the active use and exploration of a wide range of computational tools. The goal is to explore innovative architectural expressions, combine traditional culture with modern architecture, and take the plasticity of complex forms as the ultimate condition of design.

The course runs primarily using Autodesk Maya and other digital software design tools, including ZBrush, KeyShot, and Illustrator. The main design inspiration elements are limited to traditional Chinese pattern elements. The designer uses digital tools to refine and redesign them, explores their possibilities. The incredible possibilities afforded by these new technologies allow students to reinterpret the tradition of design thinking, allowing abstract ideas to be realized and new techniques applied to develop a new form of geometries, combine the new geometric forms with the existing architecture, and study the visual impact and aesthetic experience of new design. 


\subsection{Methods}

The entire education course is a process of recognizing, refining, and constantly trying of complexity. It is a process from complex to simple, and then from simple to complex (Figure 1).

- Graphical (based on drawings and sketching)

Combining with phenomenology and typology research methods. Through drawing analysis, summarizing the rules of figures and shapes as the basis of design practices.

- Analytical (based on practice)

Decomposing the whole complex to specific forms with limited variety and quantity. Exploring the regularity and variability of both visual and accurate parts. Finding the potential for architecture design.

- Empirical (based on the perception of aesthetic of actual shapes and geometries).

Making a practical attempt at the new generated patterns, replacing the abstract mathematical theory with concise diagrams, and clarifying the emotional design problem rationally. It is also a new branch of architectural design aesthetics.

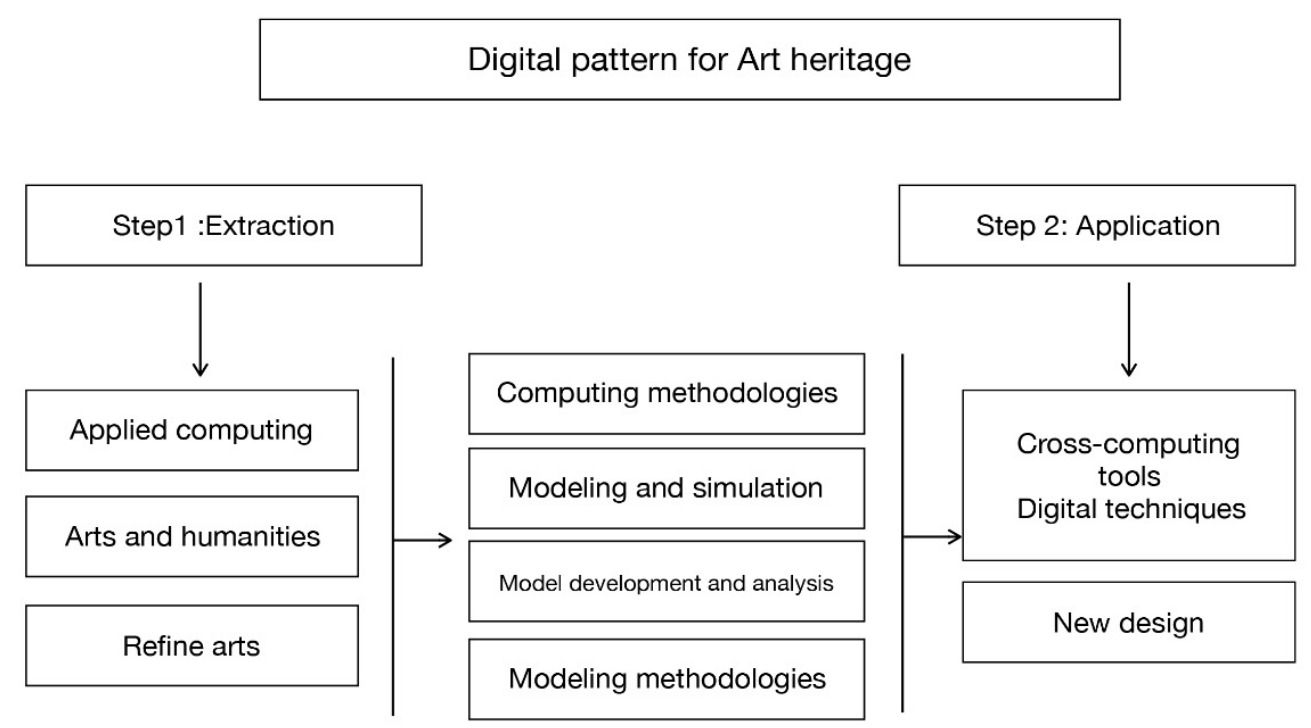

Figure 1. Diagram for method explanation.

\subsection{Extraction of the Pattern Elements}

The traditional Chinese motifs are very intricate and luxurious, with obvious signs of the times. Moreover, they did not lose the inheritance of national characteristics with the changing times. Chinese patterns also draw many advantages of foreign graphic designs. The designation of symbol patterns mainly includes two categories of abstract geometric figures (including various characters, such as oracle bone inscriptions) and natural sceneries from the perspective of the theme. Shape and color constitute the structure of pattern language. The philosophical systems of aesthetics between China and the Western countries are slightly different, so it is not appropriate to directly quote the Western theory to understand the beauty of traditional Chinese motifs. In the Chinese lifestyle, although art and aesthetic activities have specific rules, they do not only focus on possible structures and forms. It is an expression of overall judgment in resonance with nature. As Cai Yuanpei said: "Chinese painting, based on calligraphy, has the interest of literature. Western painting, related to architecture sculpture, has the scientific observation and philosophical thinking. Therefore, in Chinese painting, better with its vigor and rhythm, a painter is also good at writing and poems. In Western painting, better with skill and connotation, those who are good at painting also are skillful in architecture and drawing. Moreover, the development of art is accompanied by the development of technology" [31]. 


\subsubsection{Pattern Analysis}

Chinese traditional patterns can be divided into three types: Individual style, continuous style, appropriate style.

- Individual style

1. Symmetric

It is also called homogeneous. The patterns turn from left to right, up to down or all-around based on the hypothetical central axis or midpoint. The whole structure is rigorous and full, with neat rules. Subdivision can be divided into two forms: Absolute symmetry and relative symmetry.

Absolute symmetry: The symmetry axis or symmetry point of the patterns has the same shape and color, isometric organization, with an exact, calm, severe, and stable style, a strong sense of power. According to the different angles of symmetry, there are generally three forms: Left-right symmetry, up-down symmetry, reverse symmetry. According to the fundamental organizational dynamics, they can be divided into independent, relative, opposite, cross, centripetal, centrifugal, and combined forms.

Relative symmetry: The overall outline of the pattern is symmetrical, but there are some slightly different parts in shape or quantity with a combination freshness of dynamic and static.

\section{Balanced}

The balanced type is not limited by the symmetry axis or the symmetry point but free structure and stable center of gravity. The motif of the pattern is prominent, interspersed freely. The style is flexible and changeable. The sense of movement is strong. It can be divided into vortex form, $\mathrm{S}$ form, relative type, opposite type, cross-type, polyline type, overlapping type, and comprehensive type.

- Continuous style

\section{Two-direction continuous}

The two-direction continuity is the content of the design application. This art form can be seen everywhere in life. It is a pattern formed by a single mode (one model or a combination of two or three models), repeated on "up and down" or "left and right" direction. There are three types of continuous bone structure in two-direction: Vertical type, scattered type, and corrugated type.

\section{Four-direction continuous}

The four-direction continuity is a pattern obtained by arranging and extending one mode unit within up, down, left, and right four directions. The four-direction continuous pattern usually used for large-scale decorations, such as flower cloth, wrapping paper, wallpaper, floor tiles, etc. There are three types of bone structure in four directions: Scattered points, dislocations, and overlaps.

- Appropriate style

Appropriate patterns are rigorous and practical. The changes in patterns not only reflect the characteristics of objects but are also interspersed with nature, which forms the independent decorative beauty. The pattern is complete in shape. The internal structure and external form are cleverly combined. It is often used independently for arts and crafts decoration.

\subsubsection{Skeleton Analysis}

No matter what kind of style the patterns are, they are all formed on the corresponding skeleton structure, following a typical classics logic. First, we can summarize the rhythmic sense of the patterns with simple basic geometric shapes. These ever-changing graphic patterns are all based on the extension and expansion of a specific skeleton structure. The distribution rhythm can be quantified. Even the most complicated patterns can be subjected to modulus analysis and disassembled into delicate 
components that can be assembled and decomposed. The linking activities between elements have a general law analysis after the basis of quantitative analysis. We use drawing and mathematical methods to convert them into a computer-operable language, which becomes the basis for parameterizing graphics (Figure 2).
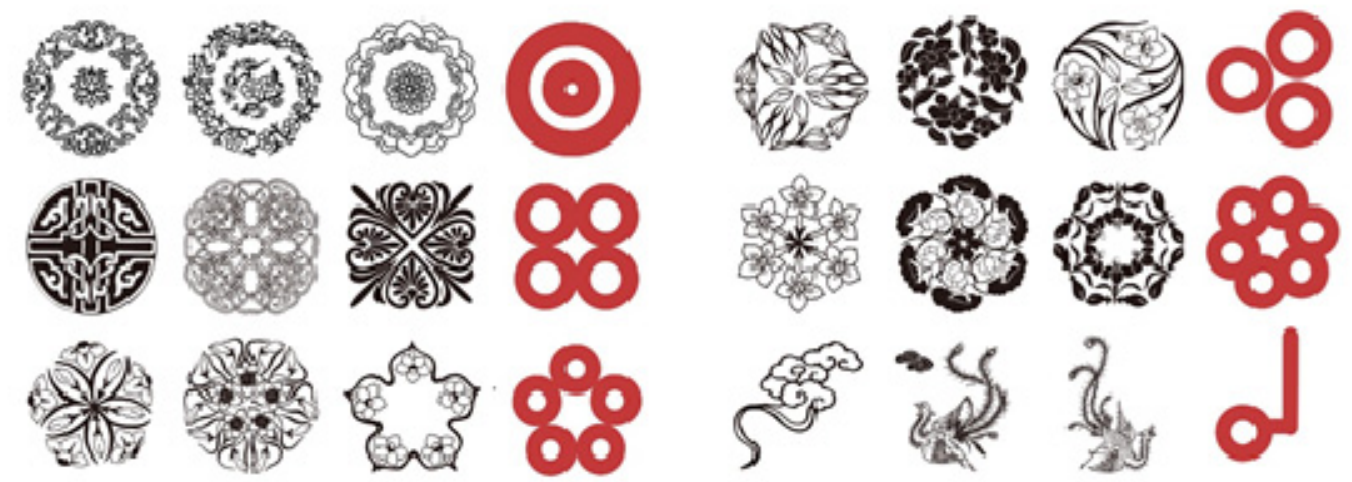

Figure 2. Some skeletons of traditional Chinese patterns.

- Taiji skeleton

The circular pattern in China is directly related to the ancient Chinese culture "Taiji" motif, a kind of graphic with an S-shaped line in a perfect circular bisect. The line divides the whole circle into two parts: The interaction between Yin and Yang, endlessly turning with a circle center.

The "Taiji" motif represents a kind of movement where the realities are complementary, as China's philosophical idea of the unity of opposites. The S-shaped line pattern is dynamic and changeable. It achieves a unique mood in its form under the support of pattern skeleton structure.

Analyze it by the coordinate axis. All activities are around a fixed center point, which is the origin. We combine the areas divided by the extended $X$ and $Y$ axes in the form of curve intersections. The pattern formed in this way is more vibrant than the layered pattern as a matryoshka. The participation of the curve can make people more visually enjoyable rather than being restricted by logic (Figure 3).

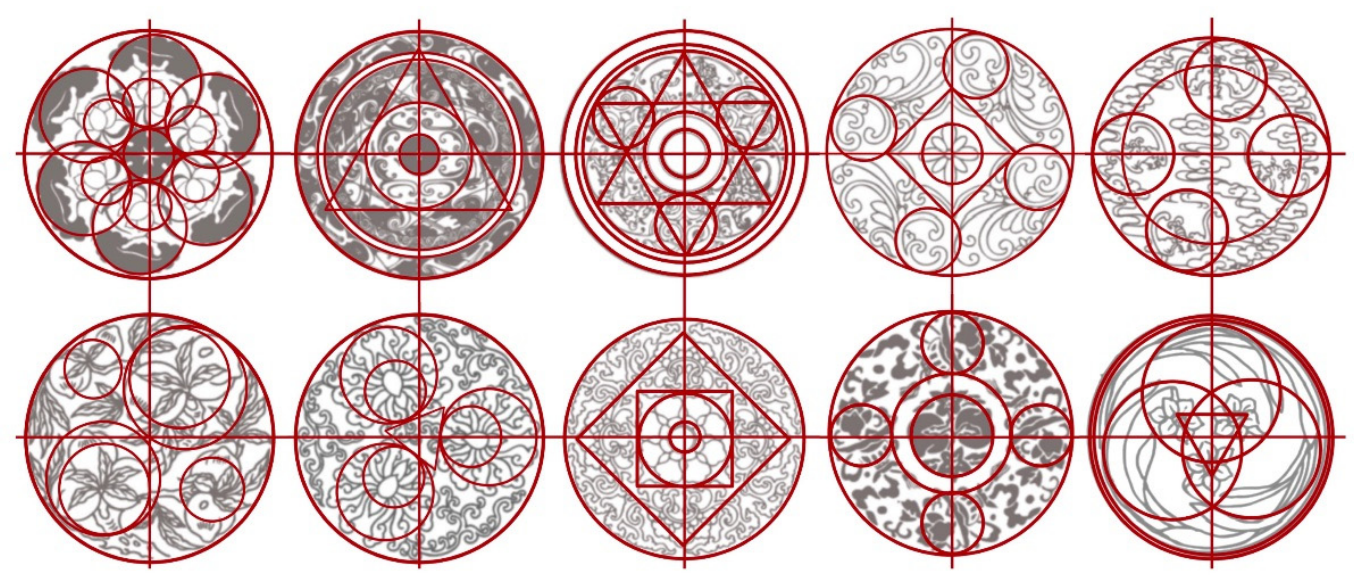

Figure 3. Circle skeleton analysis from "Taiji" motif.

- Grid skeleton

There is an old Chinese saying "everything has its counterpart". That is "Even". In Chinese tradition, "Even" is the beauty. Architecture and music are also based on the principle of "Even" as beauty. People in the "Tang" Dynasty (618-907) studied calligraphy and copied the inscriptions on a square grid. One big network can be divided into nine small grids, like the Ming (1368-1644) Emperor's 
"Jiu gong" grid of the ancient emperor. In the Qing Dynasty (1636-1911), there were thirty-six minor divisions in one grid, which was named "New Jiu gong" Style. "Jiu gong" style means: Two and four in front, six and eight in back, left three right seven, left nine right one, five in center" [32]. The diagram is arranged in order, horizontally, vertically or 45-degree Angled, all three numbers add up to 15, it is also called Magic square (Figure 4).

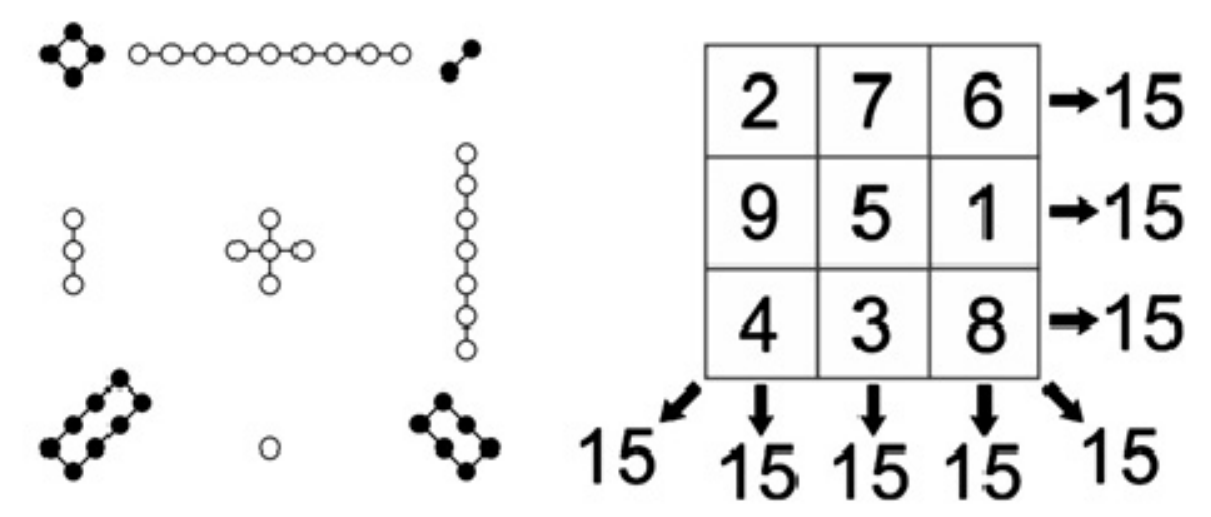

Figure 4. "Jiu gong" style.

"No rules, no standards", in Han Dynasty (206BC-220AD) it has already begun to pay attention to "rules". Like the network map pattern on the portrait brick, it contains the law and logic of mathematics as well as "symmetry", "balance", "harmony", and "proportion". The bronze mirror patterns of Han and Tang (618-907) dynasties, the composition of Han stone carvings, and the geometric patterns evolved from the "Jiu gong" all contain the unique mathematical order.

Since the Han Dynasty, there have been two "methods" in the construction of Chinese patterns. One is "four directions and eight azimuths" from the "meter-shaped grid", which based on the geometrical formation of "up and down", "left and right", "diagonal lines". This skeleton is fully reflected in the folk crafts, including batik paintings or the back of the bronze mirror. The other is the Han Dynasty stone carving composition on portrait brick and stone. The "up and down", "left and right", "diagonal lines" are combined into a three-dimensional space structure with "top surface", "side surface", and "front surface", transforming the planar composition into a three-dimensional parallel perspective pattern.

When people are trying to abstract something, they are willing to go the other way and choose some logically regular expressions that can be controlled by the human brain. Therefore, the patterns extracted by human processing have distinct structural rules. The display of traditional Chinese motifs is mostly represented on the style of two-dimensional graphics in the form of curves and natural shapes. In the complex pattern system, the essential elements interact and support each other, describing the ecological collection of sophisticated space. Before design practice, it is necessary to understand the structure order and to extract the components, the organization of the symbol of the element. These elements will bring the historical style and life vitality to the three-dimensional pattern structure. As the lines and color blocks from artists, they are full of perceptual expression. In the creative process, the designers add more efficient structural perceptual vitality components using rulers, with computers and other auxiliary tools. For example, the cloudscape in Shang (1600BC-1046BC) and Zhou Dynasties is mainly an S-shaped, T-shaped cloud pattern and hook moire pattern, in Wei (220-280) and Jin (265-420) Dynasties it appeared as a cloud pattern combined with a prominent head and tail.

\subsection{The Application of Digital Technologies}

The second step is how to apply digital technology to the protection, interpretation, and recreation of art and cultural inheritance. Reconstruct a new structure with the extracted elements by Autodesk Maya, Rhino, and Zbrush. Maya and Rhino are two commonly used modeling pieces of software for free-form shape, which respectively represent Nurbs surfaces and Polygon surfaces. Rhino modeling 
process is based on the geometry logic. The form is not limited by accuracy, complexity, order, or size. Maya software is mainly used in the film and animation field. In the design attempt, the model not only integrated the polygonal subdivision modeling technology but also combined dynamic, animation and deformation tools to generate complex structures. The specialty is that it provides a powerful polygon network modeling method, the target shape can be continuously subdivided, and the operation of different subdivision levels is essentially a geometric definition process of topology.

It is a process of topology changes and parameter changes. Besides, Maya is a modeling software with a physics engine. Due to the operation of point clouds and particle systems, physical factors can be introduced in the process of geometric construction, thereby responding to the temporality in the physical environment. Compared with Rhino, Maya can be considered as software for temporal transformation in geometric parameterization. The project experiment uses Rhino and Maya software to simulate traditional pattern illustrations, use the data relationships in the study, establish physical models, and adjust parameters as needed to provide data models for digital construction. Zbrush is a digital sculpting and painting software, widely used in the computer graphics industry to assist Maya software.

The software collaboration is to express the natural and sophisticated geometrical form of the building to the fullest. It will enable the patterns reconstructed away from the formation generated by the optimal algorithm. The essential elements can continue the Baroque aesthetic-the ultimate and luxurious aesthetic forms as far as possible. The design mainly emphasizes the sculpture style objects in the way of performance, finally ends with additive manufacturing in different scale models. Repeat the extracted essential elements by "Extrude" and "Subdivision" as two main commands. Use transformation, simulation, mutation superposition, and other methods to diversify and reorganize the elements. The entire shape, structure, and texture are all based on this. From bottom to top, from inside to outside, the accumulation method evolves a sense of hierarchy, ensuring visual extensibility and volume permeability. The form of sculpture can go beyond the basic structure. As a result of manual control, the process will not be automatically generated by software that sets up formulas or specific algorithms, but specific shapes will be carefully refined and sculpted by hand. Digital sculpture advocates an ecosystem based on the human scale. Demonstrative form organizations put individualism above the standard, digital sculpturism is loyal to its original situation, and harmoniously unifies the subject and object to make it visible. This open-minded thinking is a new way for traditional design. Designers can give full play to their potential in the production of the shapes and inspire individuals to express their desires at the maximum and the highest level in sculptural beauty, an attempt to form an extraordinary, aesthetic approach led by personal artistic concepts and visual effects. The "sculpturism" digital design methods and concepts took shape during this process.

\section{Objectives}

The paper aims to:

1. From the perspective of cultural heritage: Traditional pattern culture is an important part of Chinese traditional culture. Studying and researching its connotation is conducive to the creative transformation and innovative development of traditional culture. Look for the traditional possibilities.

2. From the perspective of design development: The design course intentions and processes deviate towards the extreme conditions in formal exploration, sustainability aesthetics, generative typologies, and geometrical, sometimes surrealistic, illusions. Look for the development possibilities.

\section{Practice Result}

Practice Subject: Take the traditional pattern as a prototype. Abstract and transform the essential elements. Combine the regenerated model with the existing old building.

The practices are based on traditional pattern graphics. First step: Confirm the mathematical logic and modulus relationship behind individual patterns and continuous patterns from its essence. Describe the relationship or structure from the accurate perspective of graphics. Complete the interpretation from 
graphics to diagrams in the design. Under digital technology, the designers try to transform complex and multi-category information into measurable numbers and data. Complex patterns are embodied, realistic, and refined into single elements which can be used for design. It is a process from complex to simple.

Then, through software processing, digital models are more sculptural. Designers use digital technology to break the standard interface orthogonal relationship of building. They try to apply shapes with free-form surfaces and irregular morphological interfaces to structures. Extremely complicated geometric forms under the control of parameters constitute a variety of visual effects with limited components. The use of these complex components in architecture makes the building more contemporary and impactful. That is, it demonstrates the value of multiple aesthetics and sociality. It is a process from simple to complex (Figure 5).

- Practice one: Floral and grass motif

1. Scroll grass pattern:

It is a model of the traditional design in floral and grass patterns. It is an exotic pattern. The scroll grass pattern in China came from the honeysuckle pattern, which was initially originated in ancient Egypt and transmitted to India via Greece. During the Eastern Han Dynasty, it was introduced into China with Indian Buddhism. After undergoing complex evolution, it eventually formed the original style in Tang (618-907). Then, a large number of ornaments were used on the sides of the stele with its concise and straightforward style and strong sense rhythm. Single-leaf mosaic, double-mosaic mosaic, or three mosaics were arranged on both sides of the trunk to form a continuous smooth two-direction continuous ribbon pattern. Scroll grass patterns have different names of different eras and regions. In Europe, it is called tendril decoration. In Arabia, it is called vine pattern. In China, it is called a scroll grass pattern or tangled pattern. In Japan, it is customarily called tang grass [33]. Therefore, it has specific Western characteristics and can be reanalyzed and transformed into computer programs based on Western design thinking (geometric analysis formula).

The scroll grass pattern is created by the extension of the S-shaped structure from the "Taiji" figure. It can be regarded as the two-direction continuous and four-direction continuous patterns formed by many secondary graphical elements. With different themes and different locations, the elemental composition changes slightly, but the details are always ordered in a ripple curve. The complete volume of the pattern has a dynamic curve grass and geometrical skeleton together. When constructing geometric bones structure, they often follow the bend in the tangential or reverse concatenation. At some links, graphics are used to attract the visual focus. The characteristics of the curl pattern are the intricate beauty of plants interlacing, simplifying the elements based on repeated observation of traditional patterns, keeping the main components of the pattern unchanged, and reducing the leaves and curls into relatively large masses. Changes in parameters have a significant effect on the role of the skeleton. Like the two-direction continuous patterns, the rhythm of the graphic distribution can be summarized by the tangent of the circular curve. The whole structure is a universal framework, and many variations can be found in specific applications. Adjust the curve value and turn fixed point vertically or horizontally. Combine the single elements in different forms to obtain variable prototypes along with different skeleton structure. Next, use the commands of rotation, copy, scaling to make the shapes prosperous. It will produce unlimited patterns with the infinite superposition of elements. The structure reaches the extreme.

The designer combined the traditional patterns and the basic shapes of flowers and plants in nature to design the final scheme form according to the growth law. Tracing the origin, there is a natural blood relationship between design and painting art. Designers extract the unique sensory characteristics of art and then use a purely rational method: Points, lines, surfaces, and blocks to form the beauty of the abstract. 


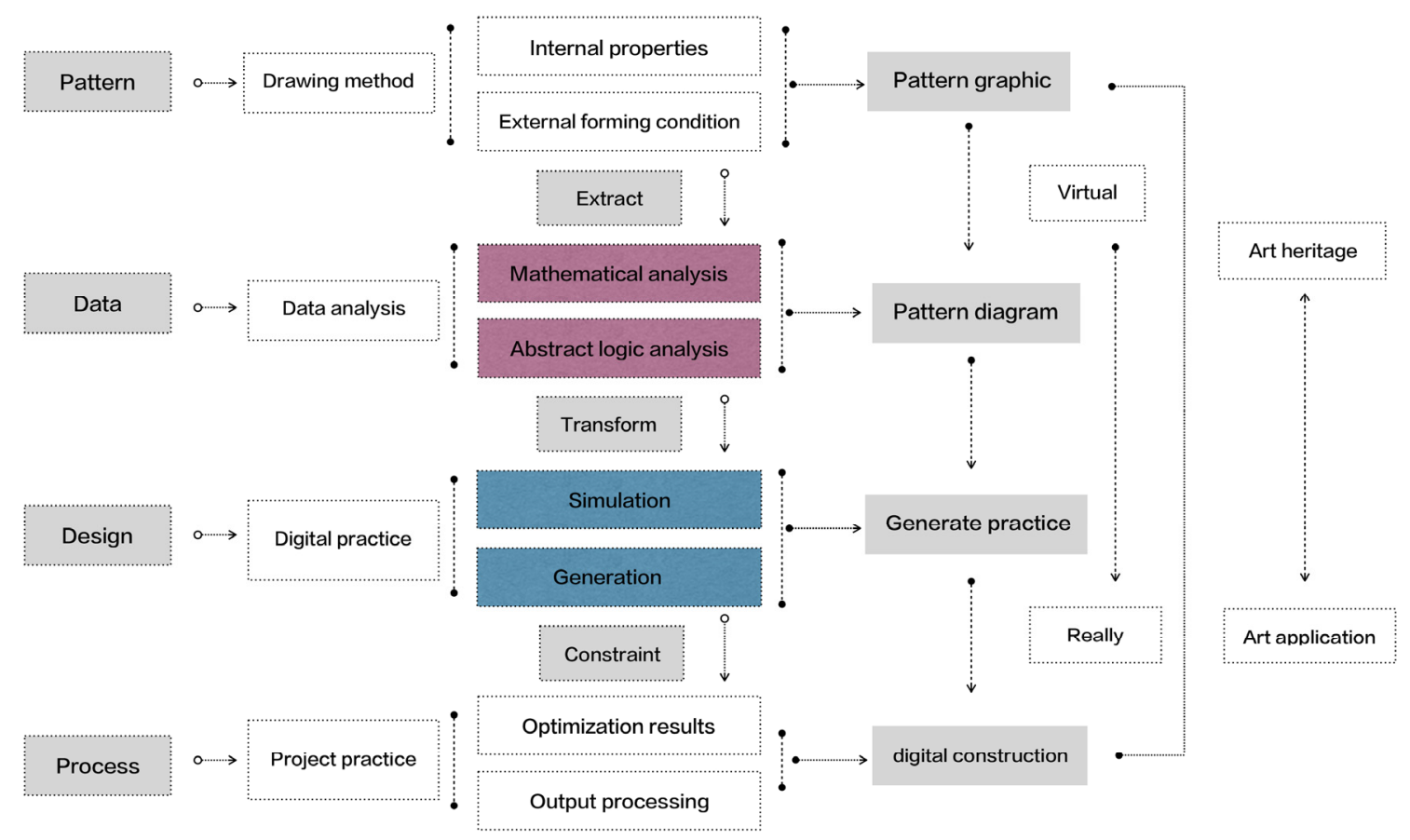

Figure 5. Explanation for the practice.

The inspiration for this practice came from the blue-and-white porcelain pattern. From the tangent and reverse changes in the figure, the designers extract the simple component from the growth of grass blades and the basic form. This single form is the basic structure that can derive the S-shaped dynamic form. Next, combine several essential elements freely to form a primary motif with further modification and optimization. After that, the final theme is generated from the primary motif with further symmetry and movement transformations, through juxtaposition, inversion, radiation, and overlap. Combine these components with existing buildings, changing the trajectory of "points" and "lines" in accordance with the original architectural form, free from the constraints of the natural way. The arc-shaped lines and body masses show a rounded, patchy, and layered sense, like flowers and grasses in nature. Attaching metal to the overall object, while pursuing nature, the design also has a sense of modernity and industrial feeling. It is a combination of nature, the utilization of natural elements, and the ultimate confirmation of modern ideas, which is a combination of nature and humanity (Figures 6 and 7).
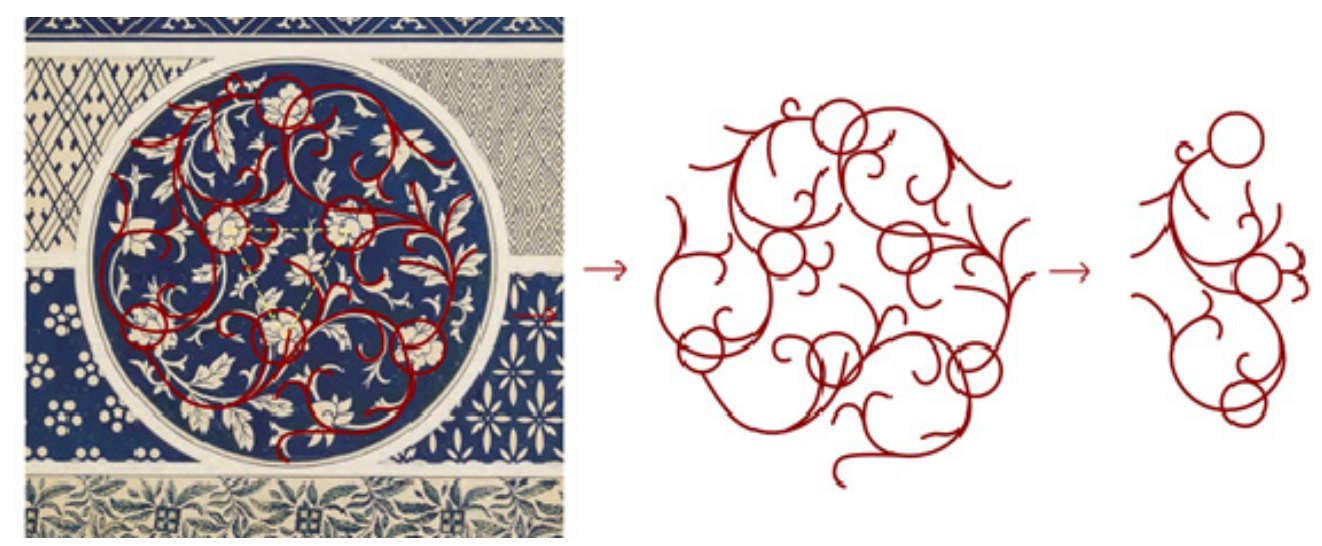

Figure 6. The analysis drawing of scroll grass pattern. 

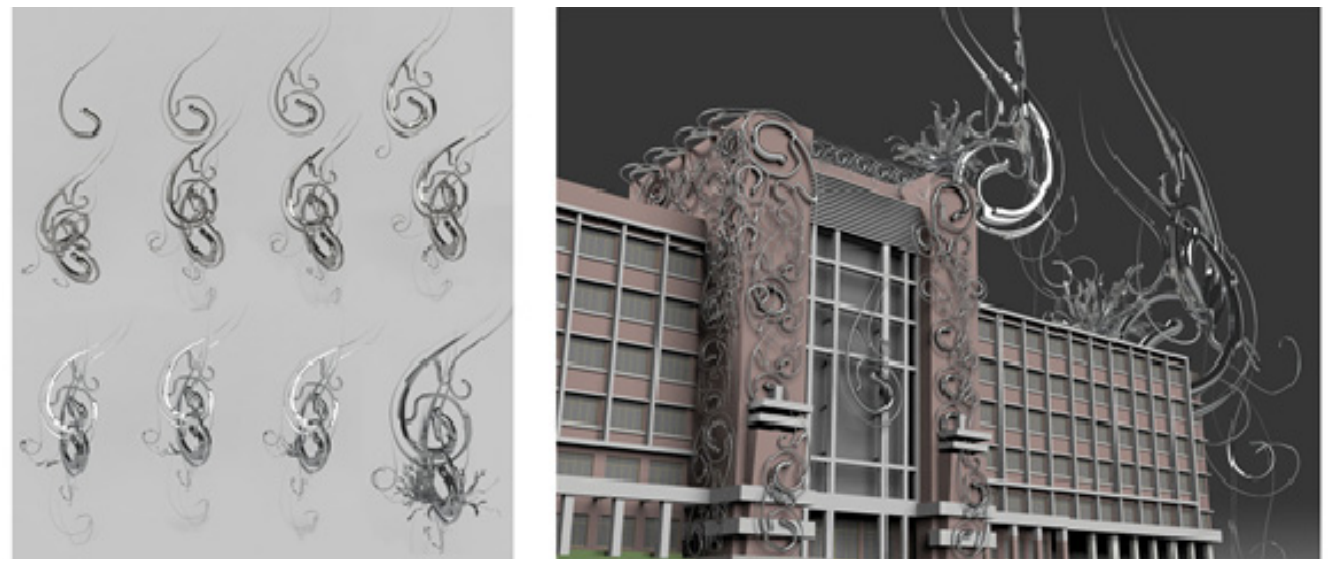

Figure 7. The application of scroll grass pattern.

The interpretation and inheritance of traditional design cannot be traced to simple repetition and simulation. It should trace its history and archetypes and move from form appearance to spirit appearance. After the shape of the entire scheme is finalized, it is further subdivided and softened. By superimposing and flipping the basic elements, the graphics go from simplicity to diversity.

- Practice two: Zoomorphic motif

Zoomorphic motifs, like a large branch of statuary art, have considerable variation and differentiation. They are the primary artistic expressions of most ethnic groups. The similarities and differences between patterns in various regions also reveal signs of influence and imitation from each other. Images and shapes have considerable changes and variations. There are multiple carriers and presentation forms, such as bronzes, tiles, lacquers, and paintings. The zoomorphic motifs, as well as the bronzewares they attached to, embody both attributes of matter and consciousness. In the early Spring and Autumn Period (771 BC-476 BC), the zoomorphic motifs shared extensive similarities, especially highlighted by the dragon motifs. In the middle of the period, new patterns were generated, like the winged lion, phoenix with dragon body, birds and beasts holding snakes in their mouths or under their feet. In the middle and late Warrior State Period, the dragon motifs in all regional systems and cultural types moved toward greater convergence.

1. Dragon pattern:

The dragon pattern is the artistic summary and sublimation of the dragon image, and it changes continuously with the development of the era until it gradually takes shape in the later period. In the Chinese inscriptions of the Shang Dynasty (1600 BC-1046 BC), there is already a precise characterization of the "Dragon" character. There are two types of fonts, traditional and simplified, all have horns, mouths, feet, and curved bodies (Figure 8).

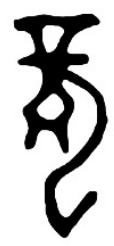

Oracle bone script

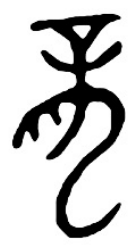

Bronzeware script

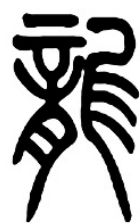

Seal script

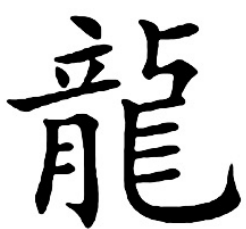

Traditional Chinese Characters

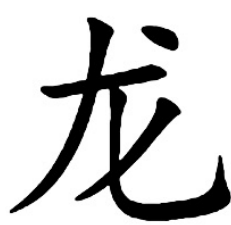

Simplified Chinese Characters

Figure 8. The development of the word "Dragon". 
The dragon pattern developed from the initial abstract chaos to the later clear contours, from a single line shape to a complex and diverse combination of divine graphics. The whole process has gone from immature prehistoric forms, the harsh and mysterious in Shang and Zhou era, the classical and simple in Qin and Han era, to the gorgeous and graceful in Sui and Tang (618-907) Dynasties, the fresh and elegant in Song (960-1279) and Yuan (1271-1368), and the complex and varied in Ming and Qing Dynasties.

This project is inspired by the dragon and phoenix pattern in the "Dunhuang" frescoes. The entire pattern spins and soars, a mood of "fly because of the wind". At the same time, the soundtrack of the dance named "Flying Apsaras" [34] also contributed to the creation of this work. The overall project is intended to show a feeling of "flying". Dunhuang's "flying" is different from the Taoist's flying in the fog, or the Western winged fly. It is reflected by the movement of clothes worn by characters. The whole building design also looks forward to the fly feeling of "satin silk grosgrain", more dynamic. The dragon motif is the starting point for the entire model element. The movement of the ribbon in the "Flying Apsaras" image is the direction of the project model. However, graphics are different from diagrams. The final form is obtained by extending the S-shaped structure. It can be considered that many secondary graphics are fluctuating along the curve and forming an intricate two-direction continuous or four-direction continuous pattern through the operations of copying, bridging, and rotating. Extend the new geometry from the length of the building and make the details appropriately large. A sensational effect, emotional visual qualities, and project details produce a thriving aesthetic (Figures 9 and 10).
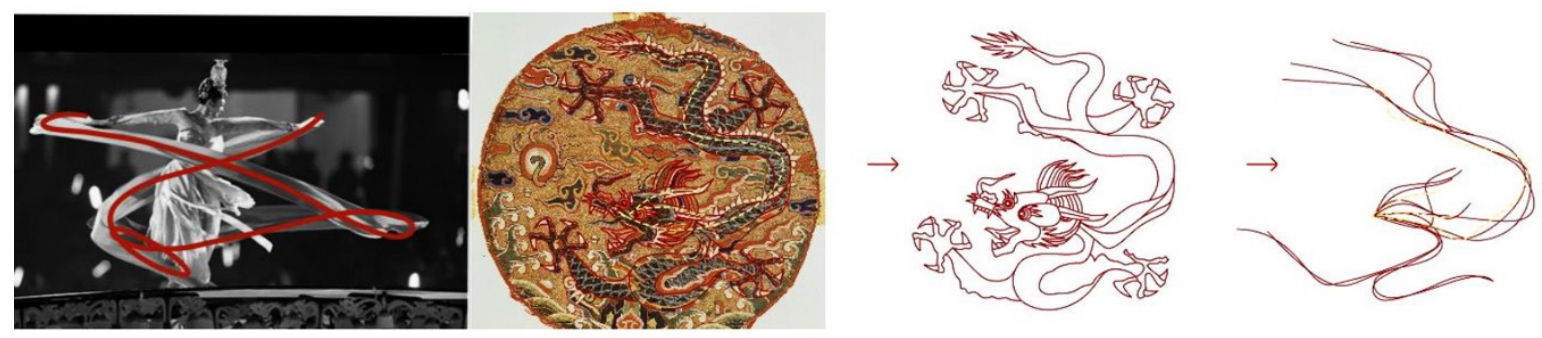

Figure 9. The analysis drawing of dragon pattern.

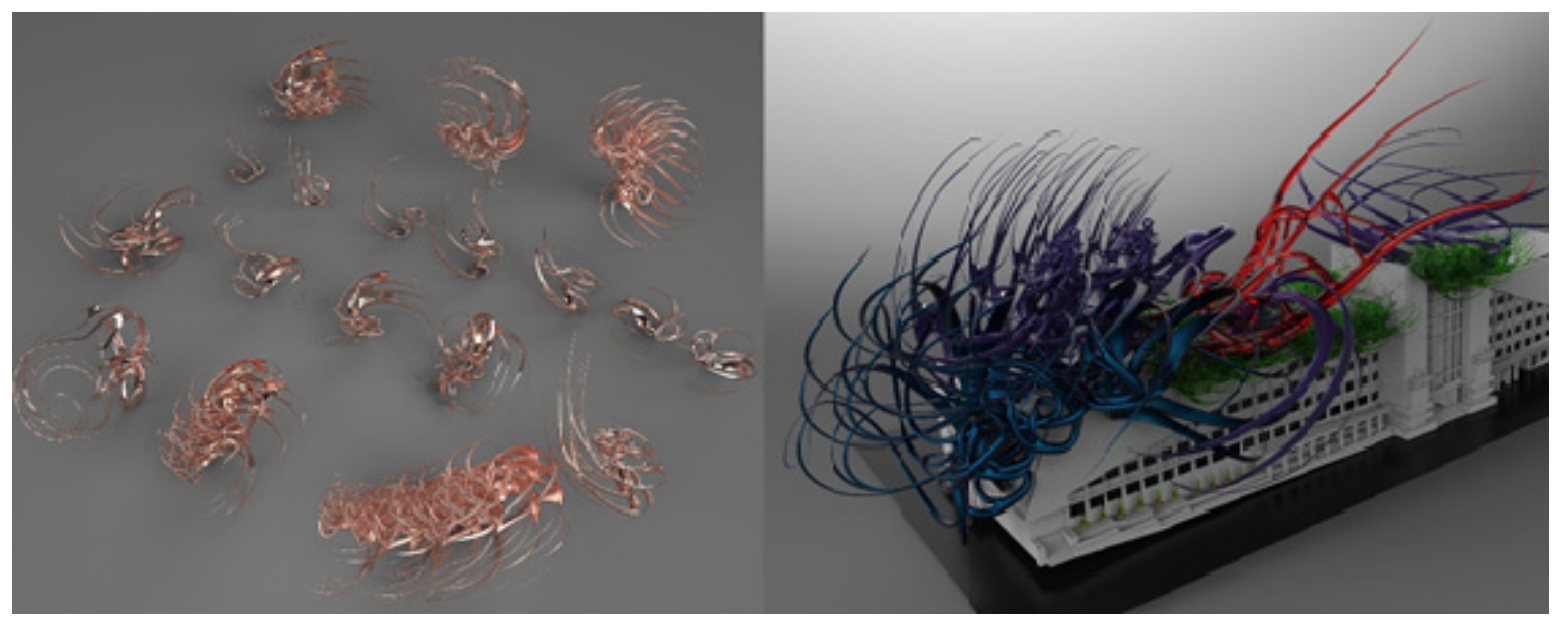

Figure 10. The application of dragon pattern. 


\section{Butterfly pattern:}

Most of the butterfly patterns exist in the field of art, and its inheritance and development have experienced the collision of ethical rules and new ideas. The application of the Chinese butterfly pattern started in the Tang Dynasty (618-907). The butterfly pattern was generally small, and it appeared in the fabric with other patterns as a decorative role. People used similar colors and shapes to describe the butterfly dancing in the fabric in pursuit of a realistic style. The pattern developed again in the Song (960-1279) and Yuan (1271-1368) Dynasties, they tended to be simple in composition, and the overall style changed from gorgeous and brilliant to elegant. The shape of the butterfly pattern was greatly influenced by the artistic style of the Song Dynasty (960-1279). It was more used to express the elegant taste of literati, but not widely used in art and fabric. In the Ming Dynasty (1368-1644), the adoption of butterfly patterns in fabrics began to show a trend of prosperity. They showed some differences among people in different classes. Among them, the upper society was pursuing sophisticated and gorgeous details. Its application showed a full development trend. There are many cases of this pattern on court dress, porcelain, and furniture in the Ming Dynasty. Although it was widely used, and the variety of designs applied in the field of art was diverse, butterfly patterns did not occupy the mainstream. In contrast, the folk butterfly pattern of this period catered to the public's aesthetic taste, and psychology pursued simplicity and generosity. For the minorities, the butterfly pattern also has a constituent symbol of culture. In history, the Miao nationality in China has no words. Patterns record the emotions and lives for the Miao in different periods through embroidery. In decorative arts, butterfly patterns are emotional and symbolic, expressing profound feelings through countless basic symbols.

The changeable shape of the butterfly pattern provides more imagination for people. When designing a single element, the designer explains the traditional butterfly pattern and finds the main part of the graphic. They use the mutation process and methodology from a cellular phase to cellular regeneration, to the system of assembly, and then the final stage of producing the "New Speciation". It works with the idea of a part to the whole relationship, from part to whole, whole becoming parts again and whole to whole, etc. It creates layers of complexity in each incremental stage of mutation. Combining the "New Speciation" with key points of the building, such as entrances, sidewalls, it has its own logic of growth and a certain kind of aesthetics (Figures 11 and 12).

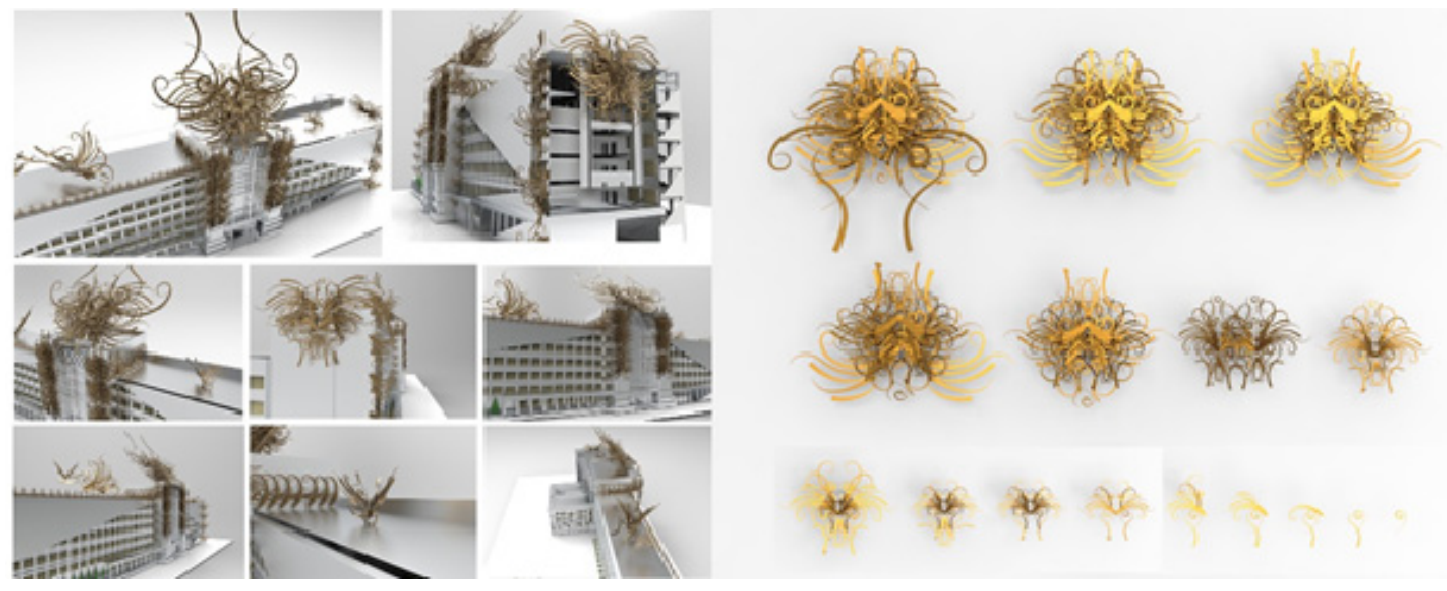

Figure 11. The analysis of butterfly pattern.

\section{- Practice three: Geometric Pattern}

Geometric patterns are a broad category of traditional patterns, appearing in the primitive society of China. It first appeared in the decoration of painted pottery in the Neolithic era. At that time, the ancestors mainly decorated their painted pottery with abstract figures, such as dots, lines, and blocks. Since then, many pattern elements on ceramics have been used, thus laying the foundation for the development of primitive art. "Various curved patterns, straight patterns, water patterns, 
swirling patterns, triangle patterns, silver tooth patterns, cloud patterns are abstract traditional geometric patterns, which are the characteristics of Neolithic pottery patterns" [35].

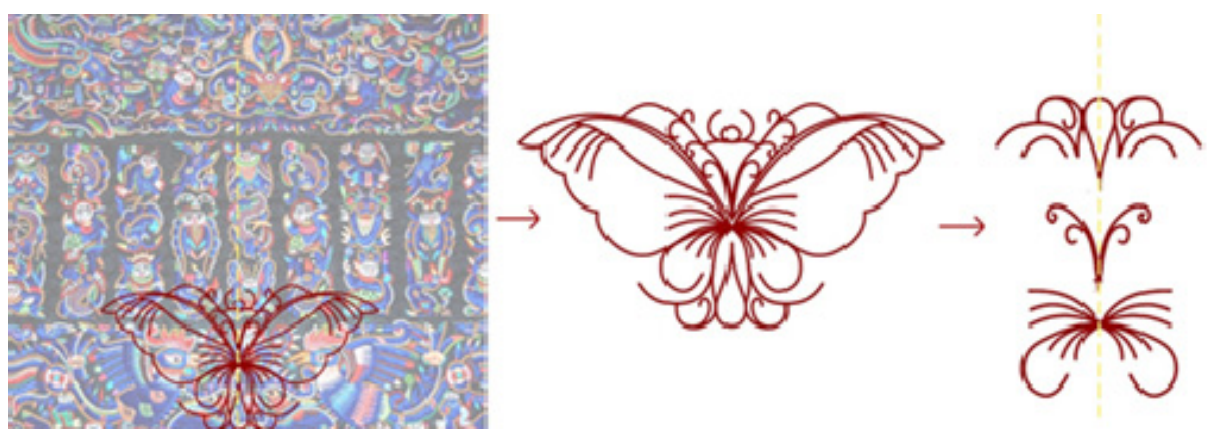

Figure 12. The application of butterfly pattern.

There are two main originations of geometric patterns: First, people hunted for a living during the primitive society. At that time, their thoughts were immature. Most of the contacts were animals and plants. Simple abstract ideas were formed by observing some natural things. It originated from the intellectual evolution of pictographic to geometric. In addition to the inevitable trend of abstraction caused by the pictographic mode of "observing objects and taking images," there are also reasons from decoration and craftsmanship. Due to the intentional or unintentional smearing at that time, the shapes formed by those points, lines, and faces slowly showed a symmetrical and regular rhythm, which gradually evolved into triangles, rhombuses, squares, and other patterns. The curves slowly evolved into patterns, such as circles and wavy lines, which are all geometric figures we are familiar with. In art, we call them geometric patterns. Second, in primitive society, people knew how to use fabrics for heating and to protect themselves with wicker weaves. The orderly beauty is shown by wicker weaving and braiding slowly formed the style of geometric decoration.

1. Cloud and thunder pattern:

Cloud and thunder pattern is a kind of geometric pattern. It is a linear form of rotation. When the linear form turns into a circle, it is called a cloud pattern. When the linear form turns into a square, it is called a thunder pattern. Although the structure of the cloud and thunder pattern also have different expression styles on various materials, they are generally expressed as photographing, bas-relief, painting, and engraving. There are also two primary forms of presentation. One is a two-direction continuous composition decorated at the central position of the container. The other is a ground pattern that fills the gaps. The overall shape is different, including diamonds, triangles, rectangles, etc. to present a full and gorgeous effect.

The inspiration for this project is a Ruyi Cloud pattern in the Qianlong period of the Qing Dynasty. The designer extracts the cloud pattern image and intercepts some of the styles from the traditional decoration, then appropriately changes and combines them to form a single body with fluency and artistic aesthetics. The individual element is generated in a discontinuous, independent, and unique manner: Some parts present a form of central symmetry, giving people a sense of balance and stability, while the other part focuses on the visual impact caused by instability force and the lively and agile image that followed. In the final combination mode, two elements are combined by copying, rotating, mirroring, and merging, then the size and thickness are adjusted. Finally, it forms a central symmetric and balanced model. In terms of material selection, the metal material is given to each monomer and the final assembly, which offers a sense of luster and smoothness, showing great visual tension pleasure (Figures 13 and 14). 

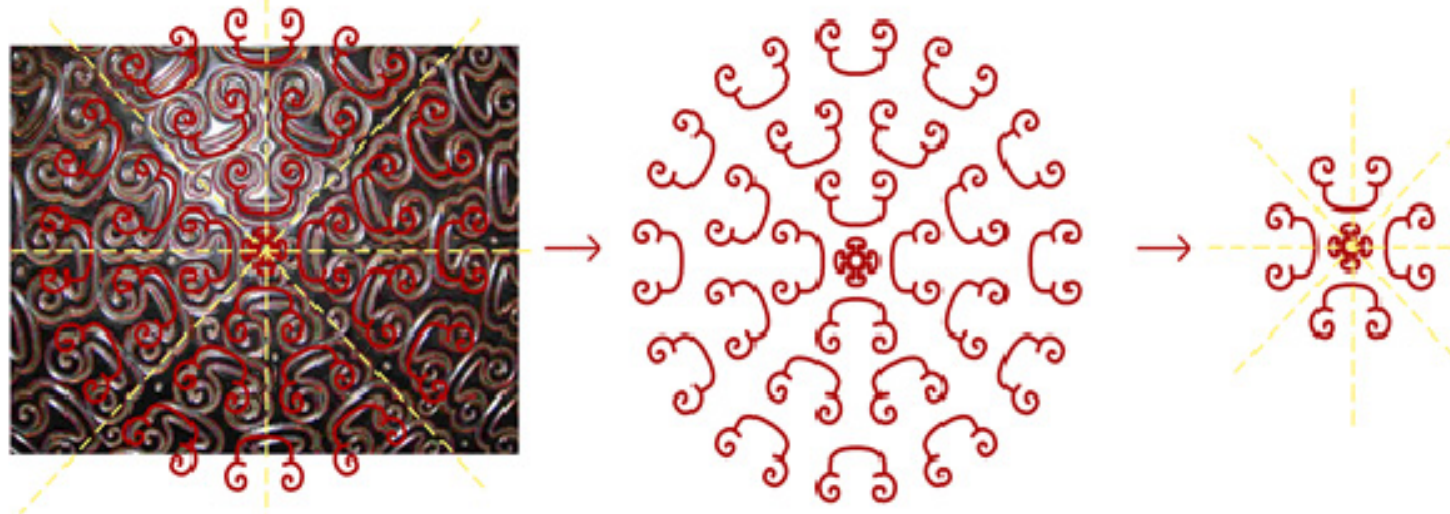

Figure 13. The analysis drawing of cloud and thunder pattern.

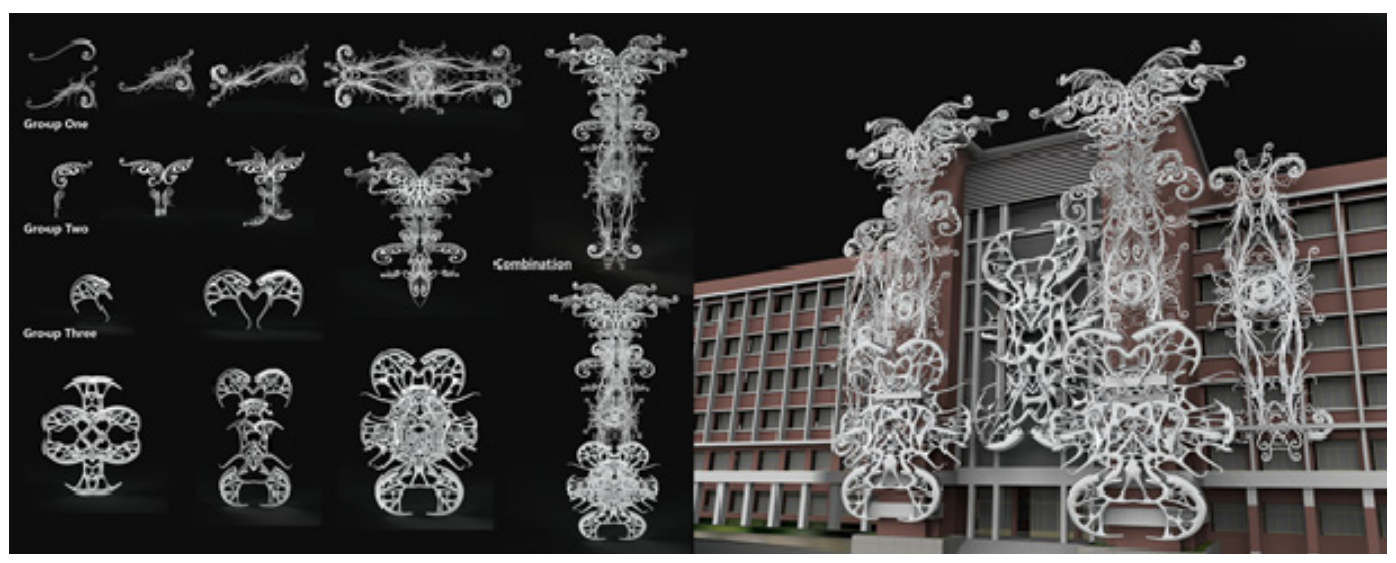

Figure 14. The application of cloud and thunder pattern.

\section{Discussion and Conclusions}

Pattern graphic design belongs to the field of art design and is often regarded as a field that depends on the right hemisphere. Digital thinking belongs to the left hemisphere of scientific rationality. They are "the front and back of a coin", one that emphasizes rationalization, and one that emphasizes the perceptual visual experience. The course abandoned the original planar application of 2D graphics, encouraged students to use digital software for multiple forms of experiments. It not only broadens the application of traditional pattern but also promotes designers to deepen the concept of "shape". Russian painter Wassily Kandinsky claimed, "The final abstract expression of every art is number. Mathematical analysis and processing of painting have enabled the art of painting to rise from perceptual to rational, from general skills to scientific or quasi-scientific status" [36]. This kind of mathematics and geometry knowledge can better explain graphic aesthetics. The more time students spend exploring the logic of the things, the more they will get and reuse. To recap:

1. In terms of design, advanced digital technology and sculpting software has changed the designer's original scope of practice and thinking method, optimized the designer's understanding and insight, and become a medium that reflects human complexity thinking. Complexity is an integration of an aesthetic desire. A tightly controlled technique is required to form transformative surfaces that incorporate distinctly different topological features, the results are potentially chaotic. Negotiations and restraining of the visual opulence of these compositions are operations that require exuberance. It represents a mutation of regeneration in the digital design process. These experimental practices brought novel sustainability and critical thinking concepts into their design processes. 
2. Inheritance in the traditional sense is written in words. The course encourages students to do reading first. Learn to respect more existence. With digital media as the carrier of traditional culture, we strive to break through the boundaries and develop new forms, new styles, and new aesthetics. Liminal form, constantly evolving the relationship between systems to create forms that are held in maximal tension in relation to one another, suspended in a permanent state of incomplete transition. This incomplete transition allows designers to recognize originality. It is like an "intermediate state" open system that allows mutations to grow indefinitely. It is an expression of aesthetic sustainability and multiple possibilities. The creative transformation and innovative development of Chinese patterns are the manifestations of the modern value of traditional culture. It is a digital inheritance, seen by more people, remembered by more people.

3. The target group of the course is the students in the sophomore year, who are not limited by a fixed thinking mode. Students can have more precise insights and a deeper understanding of new concepts. The whole process integrates the use of an aesthetic sensibility concomitant with highly developed design ability. It is a systematic thinking logic to prevent the effects of habitual processes. Students should realize that design equals energy, enthusiasm, excitement, and inspiration. To cultivate personal aesthetic ability and control ability from the elementary stage is the sustainability of education and learning.

This novel pedagogical framework explores the complete cycle of architectural design. It represents a methodical approach to "cells to organ, organs to systems, systems to new speciation", provides students with the necessary technical skills. Furthermore, it creates an experimental practice platform on which students can explore and critically apply digital technology in order to push the limits of sustainability in both the traditional culture and design education. It is a good starting point for inheriting traditional elements and finding new ideas for the design of architectural shapes. The designer tried to find new ideas for the architectural shape design, not only the reconstruction of existing design methods, but also the prediction of design methods and architectural forms that may emerge in the future.

Author Contributions: C.X. conceived the theoretical framework, designed and performed the methodology, and wrote the paper; supervision, B.D.; writing-reviewing and editing: Y.H. All authors have read and agreed to the published version of the manuscript.

Funding: This research was supported by Shandong Provincial Natural Science Foundation (ZR2018PEE023); Shandong Province Higher Educational Science and Technology Program (J18KA191).

Acknowledgments: The authors would like to thank the support of: (i) the students participating in the teaching activity, for their good interest, motivation and collaboration; (ii) the editor and the anonymous reviewers for their constructive and high level contributions.

Conflicts of Interest: The authors declare no conflict of interest.

\section{References}

1. Victor Hugo Quotes. Available online: https://www.brainyquote.com/quotes/victor_hugo_385868 (accessed on 26 April 2020).

2. Spuybroek, L. The Architecture of Continuity: Essays and Conversations; V2_ Publishing: Rotterdam, The Netherlands, 2008.

3. Ludovica Tramontin, M.J.A.D. Textile Tectonics: An Interview with Lars Spueybroek. Archit. Des. 2006, 76, 52-59. [CrossRef]

4. Galker, D.; González, A. Los Cinco Sentidos: Arquitectura y Diseño; Universidad Autónoma Metropolitana Azcapotzalco: Azcapotzalco, Mexico, 2006.

5. Rose, J. A New Sculpturalism: Contemporary Architecture from Southern California; Artforum 350 Seventh Avenue Attn: New York, NY, USA, 2013.

6. Lindsey, B.; Gehry, F.O. Digital Gehry Englische Ausgabe: Material Resistance Digital Construction; Springer Science \& Business Media: Berlin, Germany, 2001.

7. Lebbeus Woods. Available online: https:/lebbeuswoods.wordpress.com/?s=high+houses (accessed on 23 February 2010). 
8. Dehaene, M.; De Cauter, L. Heterotopia and the City: Public Space in a Postcivil Society; Routledge: Abingdon, UK, 2008.

9. Alonso, H.D.; Steele, B.; Bratton, B.H.; Petit, E.; Lan, B.Q. Xefirotarch: Excessive; HUST Press: Wuhan, China, 2007.

10. Leach, N.; Farahi, B. 3D-Printed Body Architecture; John Wiley \& Sons: Hoboken, NJ, USA, 2018.

11. Jones, O. The Grammar of Ornament: 1856; Van Nostrand Reinhold: New York, NY, USA, 1972.

12. Jones, O. The Complete Chinese Ornament: All 100 Color Plates; Courier Corporation: Chelmsford, MA, USA, 2012.

13. Graf, K.-D.; Hodgson, B.R. Popularizing geometrical concepts: The case of the kaleidoscope. Learn. Math. 1990, 10, 42-50.

14. Billings, R.W. The Power of Form Applied to Geometrical Tracery; William Blackwood and Sons: Edinburgh, UK, 1851.

15. Bain, G. Celtic Art: The Methods of Construction; Courier Corporation: Chelmsford, MA, USA, 1973.

16. Mandelbrot, B.B. Fractals: Form, Chance, and Dimension; WH Freeman: San Francisco, CA, USA, 1977; Volume 706.

17. Kharazmi, M.; Sarhangi, R. An analytical study of the methods of design and geometric constructions in architectural ornaments of the Friday Mosque of Forumad. Nexus Netw. J. 2016, 18, 275-310. [CrossRef]

18. Clévenot, D.; Degeorge, G. Ornament and Decoration in Islamic Architecture; Thames \& Hudson: London, UK, 2000.

19. Maleki, M.M.; Woodbury, R.F. Reinterpreting Rasmi Domes with geometric constraints: A Case of Goal-seeking in Parametric Systems. Int. J. Archit. Comput. 2008, 6, 375-395. [CrossRef]

20. Long, C. The Origins and Context of Adolf Loos's “Ornament and Crime”. J. Soc. Archit. Hist. 2009, 68, $200-223$. [CrossRef]

21. Schnabel, M.A. Digital Culture in Architecture: An Introduction for the Design Professions; Earthscan: London, UK, 2010.

22. Picon, A. From Tectonic to Ornament: Towards a Different Materiality. In Digital Culture in Architecture; Birkhaeuser: Basel, Switzerland, 2010; pp. 115-164.

23. Foucault, M. Space, knowledge and power (interview with Paul Rabinow). In Rethinking Architecture: A Reader in Cultural Theory; Routledge: Abingdon, UK, 1997; pp. 367-380.

24. Picon, A. Ornament: The Politics of Architecture and Subjectivity; John Wiley \& Sons: Hoboken, NJ, USA, 2014.

25. Guixiang, W. Bulletin of the Society for Research in Chinese Architecture: Its Establishment, Development and Influence. World Archit. 2016, 1, 20-25.

26. Lin, X. Symmetry and Developing Ideas: Construction-based Approach to Geometry of two-dimensional Pattern Designs. Packag. Eng. 2006, 27, 157-162.

27. 3D Printing Industry XUBERANCE'S 3D Printed Lamps Light up Milan Design Week. Available online: https://3dprintingindustry.com/news/xuberances-3d-pritned-lamps-light-milans-salone-satelliteshow-47637/ (accessed on 28 April 2015).

28. Zaha Hadid Architects Digital Turn at the Building Centre. Available online: https://www.zaha-hadid.com/ architecture/digital-turn-at-the-building-centre/ (accessed on 25 July 2018).

29. Michael Hansmeyer Computational Architecture. Available online: http://www.michael-hansmeyer.com/ digital-grotesque-II (accessed on 20 March 2017).

30. Porras Álvarez, S.; Lee, K.; Park, J.; Rieh, S.-Y.J.S. A comparative study on sustainability in architectural education in Asia-With a focus on professional degree curricula. Sustainability 2016, 8, 290. [CrossRef]

31. Yuanpei, C. Chinese People's Self-Cultivation. In Cai Yuan Pei Mei Xue Wen Xuan; Peking University Press: Beijing, China, 1983; p. 22.

32. Chen, K.X. An Interpertation of the Fourteen Types of Ancient Algorithm of Shuo shu Ji Yi: A Procedural Viewpoint. Stud. Dialect. Nat. 2003, 19, 49-54.

33. Yali, W. The Simple Comparison between Chinese and Japanese Scroll Grass Pattern. Art Des. 2009, 11. [CrossRef]

34. Flying Apsaras of Dunhuang. Available online: https://www.youtube.com/watch?v=rq3ewkItdYM (accessed on 15 February 2017).

35. Li, Z. The Path of Beauty: A Study of Chinese Aesthetics; Oxford University Press: Oxford, UK, 1994.

36. Xu, W. Drawing in the Digital Age: An Observational Method for Artists and Animators; John Wiley \& Sons: Hoboken, NJ, USA, 2012.

(C) 2020 by the authors. Licensee MDPI, Basel, Switzerland. This article is an open access article distributed under the terms and conditions of the Creative Commons Attribution (CC BY) license (http://creativecommons.org/licenses/by/4.0/). 